\title{
Influence of Temperature on Critical Fields in $\mathrm{Zn}_{x} \mathrm{Sb}_{y} \mathrm{Cr}_{z} \mathrm{Se}_{4}$
}

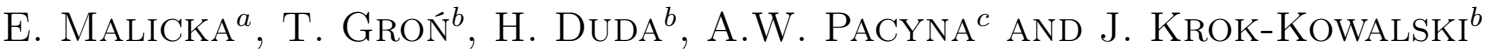 \\ ${ }^{a}$ Institute of Chemistry, University of Silesia, Szkolna 9, 40-006 Katowice, Poland \\ ${ }^{b}$ Institute of Physics, University of Silesia, Unversytecka 4, 40-007 Katowice, Poland \\ ${ }^{c}$ The Henryk Niewodniczański Institute of Nuclear Physics, Polish Academy of Sciences \\ E. Radzikowskiego 152, 31-342 Kraków, Poland
}

\begin{abstract}
The electrical and complex ac dynamic magnetic susceptibility measurements were used to study an influence of temperature on critical fields in single-crystalline $\mathrm{Zn}_{x} \mathrm{Sb}_{y} \mathrm{Cr}_{z} \mathrm{Se}_{4}$ spinel system with $y=0.11,0.16$ and 0.20 . The $p$-type conduction and a shift both of the Néel temperature $T_{\mathrm{N}}$ to lower temperatures and a susceptibility peak at $T_{\mathrm{m}}$ in the paramagnetic region - to higher temperatures were established. Below $T_{\mathrm{N}}$ the magnetic field dependence of susceptibility, $\chi_{\mathrm{ac}}(H)$, shows two peaks. First peak at the critical field $H_{\mathrm{c} 1}$ slightly decreases with temperature and remains almost constant as $\mathrm{Sb}$ content increases. Second peak at the critical field $H_{\mathrm{c} 2}$ drops rapidly with temperature and remains almost unchanged as Sb content increases. At $T_{\mathrm{N}}$ both critical fields disappear.
\end{abstract}

PACS numbers: 75.50.Pp, 75.30.Kz, 75.30.Et

\section{Introduction}

Previous structural and magnetic investigations carried out on the $\mathrm{ZnCr}_{2} \mathrm{Se}_{4}$ spinel doped with the $\mathrm{Sb}$ ions up to 0.2 located in tetrahedral sites of the spinel structure revealed the antiferromagnetic order with the Néel temperature $T_{\mathrm{N}}=22 \mathrm{~K}$ and the strong short-range ferromagnetic exchange evidenced by the large and positive value of the Curie-Weiss temperature $\theta_{\mathrm{CW}}=106 \mathrm{~K}$ [1]. The magnetic moment taken from experiment is quite close to the spin-only value for $\mathrm{Cr}^{3+}$. Chemical composition of the $\mathrm{Zn}_{x} \mathrm{Sb}_{y} \mathrm{Cr}_{z} \mathrm{Se}_{4}$ single crystals was determined by the non-destructive energy-dispersive X-ray fluorescence spectrometry showing a slight non-stoichiometry in the octahedral sites [1]. The structure refinement performed by the SHELXL-97 program package revealed that all single crystals under study have cubic structure with the space group Fd3m (No. 227). The $\mathrm{Cr}$ and ions occupy the octahedral 16e sites, while the $\mathrm{Zn}$ and $\mathrm{Sb}$ ions are in the tetrahedral $4 \mathrm{a}$ positions [1]. The structural and magnetic parameters are displayed in Table I.

\section{Experimental details}

The ac susceptibility $\chi_{\text {ac }}$ vs. external magnetic field up to $60 \mathrm{kOe}$ was measured at 4.3, 8, 12, 18, 21.2 and $22.8 \mathrm{~K}$ at internal oscillating magnetic field $H_{\mathrm{ac}}=1$ Oe with internal frequency $f=120 \mathrm{~Hz}$ using a Lake Shore 7225 ac susceptometer. The $\chi_{\text {ac }}$ vs. temperature was recorded at $H_{\mathrm{ac}}=1$ Oe with $f=120 \mathrm{~Hz}$ for external magnetic fields $H=0,5,12.5,25,40$ and $50 \mathrm{kOe}$.
TABLE I

Structural and magnetic parameters of $\mathrm{Zn}_{x} \mathrm{Sb}_{y} \mathrm{Cr}_{z} \mathrm{Se}_{4}$. $a$ and $u$ are the lattice and anion positional parameters, $R_{1}$ and $w R_{2}$ - criteria of fit, $T_{\mathrm{N}}$ is the Néel temperature, $\theta_{\mathrm{CW}}$ is the Curie-Weiss temperature, $\mu_{\mathrm{eff}}$ and $\mu_{\mathrm{s}}$ are the effective and saturation magnetic moments, respectively [1].

\begin{tabular}{l|c|c|c}
\hline \hline \multicolumn{1}{c|}{$x$} & 0.89 & 0.84 & 0.80 \\
\multicolumn{1}{c|}{$z$} & 0.11 & 0.16 & 0.20 \\
\hline$a[\mathrm{pm}]$ & 1.97 & 1.95 & 1.92 \\
$u$ & $1049.8(1)$ & $1051 .(1)$ & $1052.6(1)$ \\
$R_{1}$ & $0.25913(2)$ & $0.25925(3)$ & $0.25936(3)$ \\
$w R_{2}$ & 0.019 & 0.022 & 0.019 \\
$T_{\mathrm{N}}[\mathrm{K}]$ & 0.040 & 0.043 & 0.038 \\
$\theta_{\mathrm{CW}}[\mathrm{K}]$ & $22.0(4)$ & $21.7(2)$ & $21.8(3)$ \\
$\mu_{\mathrm{s}}\left[\mu_{\mathrm{B}} / \mathrm{Cr}^{3+}\right]$ & $108(1)$ & $106(1)$ & $103(1)$ \\
$\mu_{\mathrm{eff}}\left[\mu_{\mathrm{B}} / \mathrm{Cr}^{3+}\right]$ & $2.90(2)$ & $2.83(2)$ & $2.64(3)$ \\
& $3.81(2)$ & $3.81(3)$ & $3.84(2)$
\end{tabular}

The electrical measurements have been done in the temperature range $270-500 \mathrm{~K}$ and in the $\langle 001\rangle$ direction. The electrical conductivity $\sigma(T)$ has been measured with the aid of the 4-point dc method. The maximal error $\delta \sigma / \sigma$ was less than $\pm 1 \%$. The thermopower $S(T)$ was measured with a differential method using the temperature gradient $\Delta T$ of about $2 \mathrm{~K}$. The accuracy of $S(T)$ was better than $3 \mu \mathrm{V} / \mathrm{K}$. 


\section{Results and discussion}

The electrical results presented in Figs. 1 and 2 show the thermally activated $p$-type $\mathrm{Zn}_{x} \mathrm{Sb}_{y} \mathrm{Cr}_{z} \mathrm{Se}_{4}$ semiconductors of the Arrhenius type. A general decrease of thermopower below $400 \mathrm{~K}$ as well as an increase of the $p$-type electrical conductivity as the Sb content $y$ increases seem to be connected both with a hole transport in the lowest $\mathrm{Cr}^{3+} t_{2 \mathrm{~g}}^{2} e_{\mathrm{g}}^{0}$ band and an activation of the holes in the valence band to the acceptor energy levels. The nature of acceptor levels can be related to the Cr vacancies, occupying the octahedral sites, because a slight increase of non-stoichiometry as the Sb content $y$ increases is observed (see Table I).

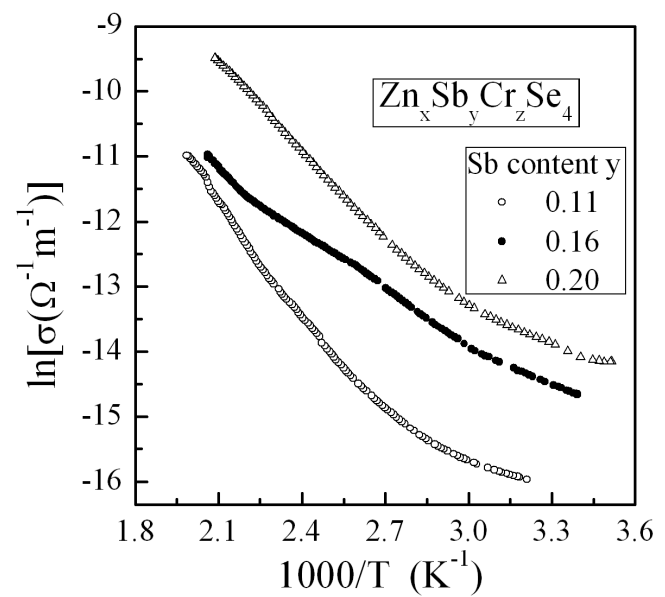

Fig. 1. The electrical conductivity $(\ln \sigma)$ vs. reciprocal temperature $T^{-1}$ for single crystals with $y=0.11,0.16$, and 0.20 of the $\mathrm{Zn}_{x} \mathrm{Sb}_{y} \mathrm{Cr}_{z} \mathrm{Se}_{4}$ spinel system.

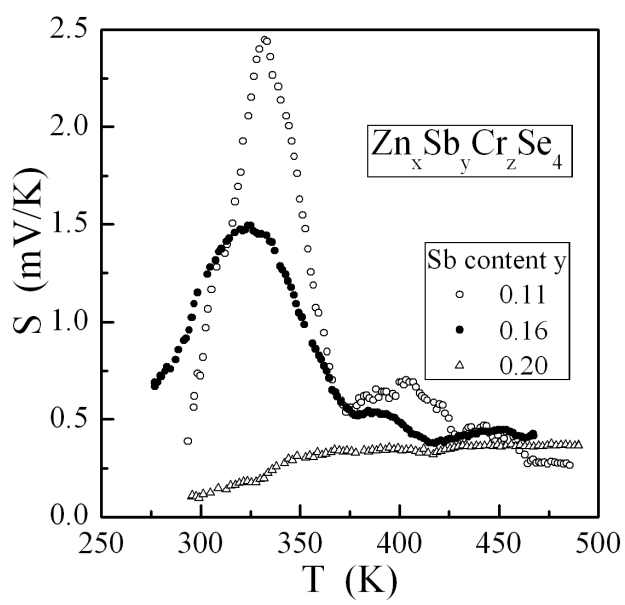

Fig. 2. The thermoelectric power $S$ vs. temperature $T$ for single crystals with $y=0.11,0.16$, and 0.20 of the $\mathrm{Zn}_{x} \mathrm{Sb}_{y} \mathrm{Cr}_{z} \mathrm{Se}_{4}$ spinel system.

The magnetic results depicted in Figs. 3 and 4 and in Table II show the strong long-range antiferromagnetic and short-range ferromagnetic interactions evidenced by a shift of $T_{\mathrm{N}}$ to lower temperatures and a susceptibility

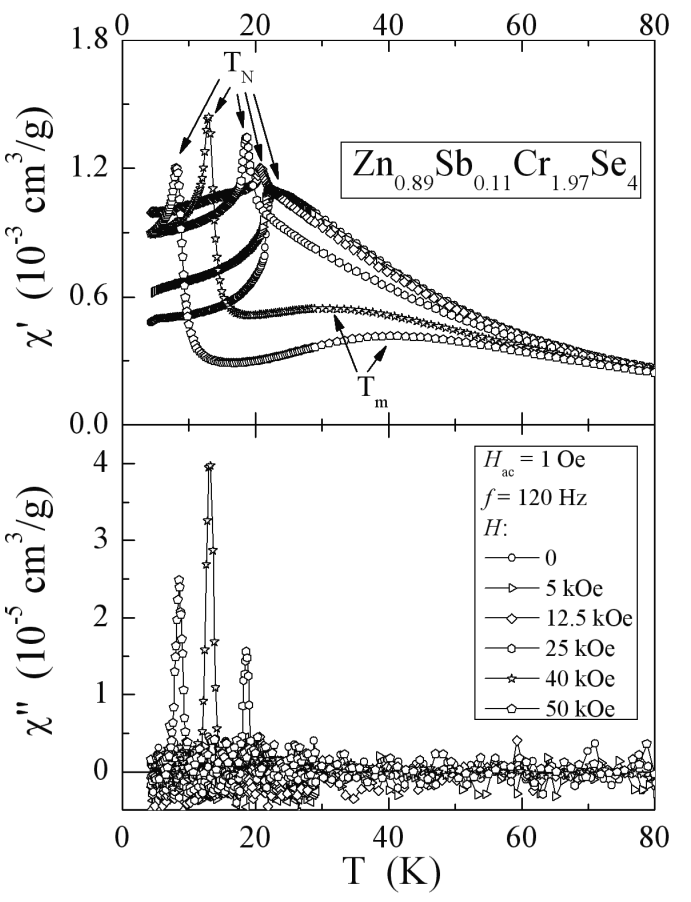

Fig. 3. In phase $\chi^{\prime}$ and out of phase $\chi^{\prime \prime}$ components of fundamental susceptibility vs. temperature $T$ for the $\mathrm{Zn}_{0.89} \mathrm{Sb}_{0.11} \mathrm{Cr}_{1.97} \mathrm{Se}_{4}$ spinel recorded at $H_{\mathrm{ac}}=1 \mathrm{Oe}$ with $f=120 \mathrm{~Hz}$ for external magnetic fields $H=0,5$, $12.5,25,40$ and $50 \mathrm{kOe}$. The Néel $T_{\mathrm{N}}$ and $T_{\mathrm{m}}$ temperatures are indicated by arrows.

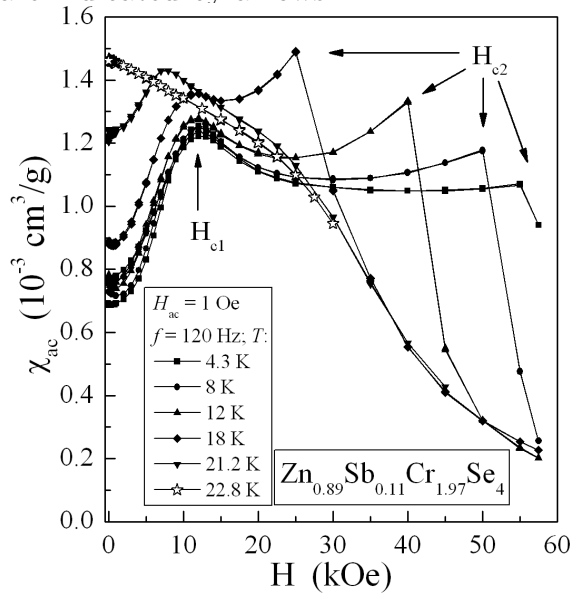

Fig. 4. Ac mass susceptibility $\chi_{\mathrm{ac}}$ vs. external magnetic field $H$ for $\mathrm{Zn}_{0.89} \mathrm{Sb}_{0.11} \mathrm{Cr}_{1.97} \mathrm{Se}_{4}$ recorded at internal oscillating magnetic field $H_{\mathrm{ac}}=1$ Oe with internal frequency $f=120 \mathrm{~Hz}$ for $T=4.3,8,12,18,21.2$ and $22.8 \mathrm{~K}$. The critical fields $H_{\mathrm{c} 1}$ and $H_{\mathrm{c} 2}$ are indicated by arrows.

peak at $T_{\mathrm{m}}$ in the paramagnetic region - to higher temperatures as a magnetic field increases, respectively. The $\chi_{\mathrm{ac}}(H)$ dependence reveals two peaks below $T_{\mathrm{N}}$. First at the critical field $H_{\mathrm{c} 1}$, connected with a metamagnetic transition and the breakdown of the helical spin structure $[2,3]$, which slightly decreases with temperature. Second at the critical field $H_{\mathrm{c} 2}$, connected with the breakdown of the conical spin structure [3], which drops strongly 
TABLE II

Critical fields $H_{\mathrm{c} 1}$ and $H_{\mathrm{c} 2}$ at 4.3, 8, 12, 18, 21.2 and $22.8 \mathrm{~K}$ of the $\mathrm{Zn}_{x} \mathrm{Sb}_{y} \mathrm{Cr}_{z} \mathrm{Se}_{4}$ single crystals for $y=0.11$, 0.16 and 0.20 .

\begin{tabular}{c|c|c|c}
\hline \hline \multirow{2}{*}{$\begin{array}{c}\text { Critical } \\
\text { fields }\end{array}$} & \multicolumn{3}{|c}{ Sb content $y$} \\
\cline { 2 - 3 } & 0.11 & 0.16 & 0.20 \\
\hline$H_{\mathrm{c} 1}[\mathrm{kOe}]$ & 12 & 13 & 12 \\
$H_{\mathrm{c} 2}[\mathrm{kOe}]$ & 55 & 57.5 & 55 \\
\hline \multicolumn{4}{c}{$T=8 \mathrm{~K}$} \\
\hline$H_{\mathrm{c} 1}[\mathrm{kOe}]$ & 12 & 13 & 11.5 \\
$H_{\mathrm{c} 2}[\mathrm{kOe}]$ & 50 & 50 & 45 \\
\hline \multicolumn{4}{c}{$T=12 \mathrm{~K}$} \\
\hline$H_{\mathrm{c} 1}[\mathrm{kOe}]$ & 12 & 12.5 & 11 \\
$H_{\mathrm{c} 2}[\mathrm{kOe}]$ & 40 & 40 & 40 \\
\hline \multicolumn{4}{c}{$T=18 \mathrm{~K}$} \\
\hline$H_{\mathrm{c} 1}[\mathrm{kOe}]$ & 11.5 & 12 & 12 \\
$H_{\mathrm{c} 2}[\mathrm{kOe}]$ & 25 & 30 & 25 \\
\hline \multicolumn{5}{c}{$T=21.2 \mathrm{~K}$} \\
\hline$H_{\mathrm{c} 1}[\mathrm{kOe}]$ & 7.5 & 11 & 8 \\
$H_{\mathrm{c} 2}[\mathrm{kOe}]$ & 0 & 0 & 0 \\
\hline
\end{tabular}

with temperature. The $H_{\mathrm{c} 2}$ is mainly responsible for a spin frustration of the re-entrant type confirmed by the experimentally observed splitting of the zero-field-cooled and field-cooled susceptibilities [1]. Both critical fields do not significantly depend on $\mathrm{Sb}$ content in a sample and they disappear at $T_{\mathrm{N}}$. The non-zero values of out of phase component of susceptibility at $T_{\mathrm{N}}$ visible in Fig. 3 seem to be associated with an energy loss connected only with the spin re-arrangement and strong long-range interactions.

\section{Acknowledgments}

This work is funded from science resources for years 2007-2009 as a research project No. N N204 178433.

\section{References}

[1] E. Malicka, A. Waśkowska, J. Heimann, T. Mydlarz, R. Sitko, D. Kaczorowski, J. Solid State Chem. 181, 1970 (2008).

[2] R. Kleinberger, R. de Kouchkovsky, C.R. Acad. Sci. Paris Ser. B 262, 628 (1966).

[3] J. Hemberger, H.-A. Krug von Nidda, V. Tsurkan, A. Loidl, Phys. Rev. Lett. 98, 147203 (2007). 